\title{
Correspondence
}

\section{Acute gout during hypouricaemic therapy: prophylaxis with colchicine}

Sir, The efficacy of the uricosuric diuretic agent tienilic acid in the treatment of both gout and hypertension is confirmed in 2 papers. ${ }^{12}$ During our inpatient study 3 of the 4 patients suffered attacks of gout, as is often the case when starting other kinds of hypouricaemic therapy. It is commonly held that the concomitant use of colchicine prevents such attacks, and accordingly we looked into this in a long-term outpatient study. Twenty patients were given tienilic acid alone and 10 patients were given tienilic acid together with colchicine $1 \mathrm{mg}$ b.d. on a nonselective basis. The patients were followed for between 2 and 12 months, with encouraging results in lowering both the blood pressure and serum uric acid, but owing to reports from elsewhere of hepatotoxicity the study had to be abandoned. Of the patients who received tienilic acid alone 15 suffered 27 attacks of gout within 2 months of starting therapy, whereas none of the 10 patients who received colchicine in addition suffered an attack of gout over the same period.

This gives support to the unsubstantiated belief that colchicine prevents the attacks of gout which are seen after starting hypouricaemic therapy.

P. Hollingworth, J. A. Reardon and J. T. ScotT Kennedy Institute of Rheumatology, London W6. Charing Cross Hospital, London W6.

\section{References}

1 Reardon J A, Scott J T. Controlled inpatient study of tienilic acid in treatment of gout and hypertension. Ann Rheum Dis 1980; 39: 367-72.

2 Gibson T, Rodgers V A, Potter C F, Simmonds H A. Tienilic acid: a single treatment for hyperuricaemia and hypertension? Ann Rheum Dis 1980; 39: 373-6.

\section{Standardisation of tests for antinuclear antibody}

Sir, The sensitivity of immunofluorescence (IF) tests for antinuclear antibody (ANA) is affected not only by the variables inherent in the IF procedure itself, but also by qualitative variations in the antigen substrate employed and the immunochemical features of antibody detected. Use of an agreed test system, however, even if practicable, would have inhibited many new observations in the field of antinuclear serology and recognition of their clinical associations. The clinical significance of reports issued by routine laboratories has thus usually been determined in the light of local experience. This incomparability between laboratories leads to difficulty in the interpretation of results obtained on individual patients tested in more than one centre and in the assessment of reported research findings.

As long ago as 1970 the World Health Organisation made available an International Reference Preparation $(66 / 233)$ for homogeneous 'antinuclear factor' on the basis of an international collaborative study which ascribed to this material a potency value of 100 international units. It was shown that its use as an external standard leads to satisfactory comparability between different laboratories in tests on the same sera. The preparation is available on application in this country to the Director, National Institute for Biological Standards and Control, Holly Hill, Hampstead, London NW3 6RB, and internationally from The Director, WHO International Laboratory for Biological Standards, Statens Seruminstitut, 80 Amager Boulevard, DK 2300, Copenhagen S, Denmark. Precise instructions for using the material as a potency standard are issued with the preparation.

Thus we should no longer tolerate modes of reporting ANA levels exemplified at a recent Heberden Society meeting where in 3 papers ANA results were communicated as 'positive' or 'negative', or as titres, without reference to the sensitivity of the methodology used. We therefore respectfully suggest that speakers at Society meetings express their ANA findings in international units and further, Sir, that the Annals of the Rheumatic Diseases sets an example by requiring authors of manuscripts reporting ANA results to do the same.

\section{G. D. JoHnson AND E. J. HolboRow Bone and Joint Research Unit, London Hospital Medical College, 25-29 Ashfield Street, London E1 2AD}

\section{Gold binding to blood cells}

Sir, Van de Stadt and Abbo-Tilstra ${ }^{1}$ reported the binding of gold to blood cells and serum proteins during aurothioglucose therapy in patients with rheumatoid arthritis (RA). The authors state that the only other team of investigators to examine blood cell gold distribution in vivo was Ward et al. ${ }^{2}$ However, Freyberg et al. ${ }^{3}$ in 1941 found 'all the gold in blood was found to be present in the plasma', and Coke ${ }^{4}$ was unable to demonstrate gold uptake in circulating leucocytes. In 1961 Lawrence $^{5}$ administered intramuscular ${ }^{198} \mathrm{Au}$ gold sodium thiomalate to $10 \mathrm{RA}$ patients, 7 of whom were currently receiving chrysotherapy. The red cell gold concentration was approximately one-quarter of the plasma level the day following injection and fell less rapidly than the plasma level. In 1973, using ${ }^{195} \mathrm{Au}$ labelled gold sodium thiomalate, our group 6 reported that $40 \%$ of 16 RA patients had measurable quantities of gold in the blood cell mass and in isolated red blood cells. This figure corresponds closely to the $45 \%$ occurrence of blood cell gold observed by Van de Stadt and Abbo-Tilstra. Other similarities between our data and Van de Stadt and Abbo-Tilstra's include: (1) the quantity of gold bound to the cells (up to $45 \%$ and $35 \%$, respectively); (2) the lack of correlation between serum gold concentrations 